\title{
Visual Pathway Alterations after Severe Acute Occupational Elemental Mercury Poisoning: Report of a Series of 29 Cases
}

\author{
Salvador Pastor-Idoate \\ Hospital Clínico Universitario de Valladolid \\ Rosa M Coco-Martin ( $\square$ rosa@ioba.med.uva.es) \\ University of Valladolid https://orcid.org/0000-0002-1811-1417 \\ Iratxe Zabalza \\ Hospital Puerta del Mar: Hospital Universitario Puerta del Mar \\ Yrvani Lantigua \\ Hospital Universitario de Burgos \\ Itziar Fernández \\ University of Valladolid \\ Jose Luis Pérez-Castrillón \\ University of Valladolid \\ Ruben Cuadrado \\ University of Valladolid \\ Jose Alberto de Lazaro \\ University of Valladolid \\ Angela Morejon \\ IOBA: Instituto de Oftalmobiologia Aplicada \\ Antonio Dueñas-Laita \\ University of Valladolid \\ Jose Carlos Pastor \\ University of Valladolid
}

Research

Keywords: Mercury poisoning, Mercury vapor, Occupational exposure, Optical coherence tomography (OCT), Ocular Electrofisiology

Posted Date: November 25th, 2020

DOI: https://doi.org/10.21203/rs.3.rs-113238/v1

License: (c) (1) This work is licensed under a Creative Commons Attribution 4.0 International License. Read Full License 


\section{Abstract}

BACKGROUND: There are almost no available data on retinal involvement after acute exposure to high concentrations mercury and the available reports are on a small number of patients that suffered chronic expposure. The purpose of this paper is to report visual findings in 29 workers exposed to very high concentrations of mercury vapor in a factory in northern Spain in 2012.

METHODS: Twenty-nine patients and 16 controls were evaluated in a comparative case series. Fifteen of the 29 workers underwent belated chelation for heavy metal intoxication, only 3 in a prompt way. The mercury levels in blood and urine samples, visual acuity (VA), contrast sensitivity (CS), visual field (VF), color discrimination and optical coherence tomography (OCT) data were recorded. The pattern reversal visual-evoked potentials (PRVEP), full-field and multifocal electroretinography (ffERG/mfERG), pattern electroretinography (PERG), systemic symptoms, presence of erethism, and electromyography (EMG) were also gathered. A descriptive analysis was performed. The evolution of patients who did not undergo chelation (group 1), those who underwent late chelation (group 2), and those with deep VF defects (group 3) were compared with a control group. The correlations between variables also were studied.

RESULTS: The VA was affected slightly. The loss of CS in at least one of four spatial frequencies and color vision alterations occurred in $96.5 \%(n=28)$ and $44.8 \%(n=13)$, respectively, in the entire group. VF alterations were identified in $72.4 \%(n=21)$. No morphologic changes were seen in the OCT scans. Latencies over 100 milliseconds and reduced amplitudes of P100 were found in the PRVEP. The ffERG and PERG results suggested that both the outer and inner retinal processes were involved. The mfERG indicated reduced parafoveal retinal function. Twenty-six workers exhibited symptoms of erethism. The EMG showed sensorimotor polyneuropathy and multiple mononeuropathy alterations. Significant negative correlations among blood mercury levels, VA, and ffERG were observed.

CONCLUSION: Advanced visual functions were impaired significantly independent of the mercury levels. Delayed chelation was not beneficial. Although neurologic and visual pathway involvement was clearly demonstrated, this study also showed clear functional retinal participation in impaired vision.

\section{Background}

Acute or subacute poisonings as the result of exposure to elemental mercury in the workplace are uncommon. However, these episodes may have profound effects on the visual pathway due to the neurotoxicity of this metal [1,2]. Controversy exists on the magnitude of the possible damage in the retinal structures. Some experimental studies have reported the presence of mercury in the retina and choroid after systemic administration $[3,4]$, but others have limited the presence of the metal to the retinal pigment epithelium and external neuroretinal layers $[5,6]$.

The introduction of electrophysiology tests and current clinical examination techniques such as autofluorescence or optical coherence tomography (OCT) have suggested that besides central nervous system (CNS) poisoning, which is widely accepted, the retina also is involved and that not all visual functional alterations are caused by high visual pathway damage [6-8].

Although physiologic and morphologic retinal changes resulting from mercury toxicity have been demonstrated in animal models; there are few reports of human retinal effects resulting from occupational poisoning in the literature; the last long series on mercury poisoning in humans was published before the latest retinal diagnostic techniques, i.e., spectral-domain OCT (SD-OCT) or multifocal electroretinography (mfERG), became available in clinic. Only one group has published OCT studies but on patients who had been exposed chronically $[7,8]$, and only one study has been published on mfERG results in patients with color vision loss who also were exposed chronically to mercury [6]. To our knowledge, the current study is the first that includes both techniques and pattern ERG (PERG) to evaluate morphologic and functional retinal findings after acute exposure.

In the current study, we describe the visual pathway alterations in 29 workers inadvertently exposed to very high concentrations of mercury vapor for 14 consecutive days during maintenance work in a factory in northern of Spain at the end of 2012, one of the most severe incidents of acute elemental mercury intoxication that occurred in the European Union.

The opportunity to study those patients and the improvements in the retinal evaluation techniques have provided essential information on the degree of the mercuric effects at the intraocular level.

\section{Methods}


This comparative case series followed the tenets of the Helsinki Declaration of 1964 (last amendment, 2013). The Clinical Research Ethics Committee of the Valladolid East Health Area approved the study and patients provided written informed consent.

\section{Patients}

According to official company sources, 49 patients were exposed inadvertently to elemental mercury vapor while performing maintenance work in a heat exchanger. The incident occurred from November 19 to December 2, 2012, in a metal manufacturing plant in northern Spain. According to the workers' accounts, upon entering the workspace, they observed balls of mercury spread over the floor. A few days after finishing the work, many workers presented with physical complaints that included asthenia, headache, lumbago, cough, bitter taste, dental pain, gum inflammation and bleeding, and epigastrium and abdominal pain among other symptoms, which was attributed initially to a viral infection.

After this initial symptomatology, most patients began to develop mercury-related erethism including fatigue, irritability, aggressiveness, anxiety, depression, and insomnia and neurologic manifestations that included tremor, peripheral polyneuropathy, weakness, headache, cognitive disorder, dizziness, and digestive manifestations such as diarrhea and abdominal cramps. Many also presented with visual complaints of blurred vision, ocular irritation, dry eye, burning or scratchy sensation, eye redness, and light sensitivity.

The levels of mercury in blood and urine, measured from the second week of the exposure, exceeded the biologic limits recommended for occupational exposure $[9,10]$, with some levels between 500 and $900 \mathrm{ug} / \mathrm{L}$ in blood and between 600 and $1,830 \mu \mathrm{g} / \mathrm{g} \mathrm{Cr}$ in urine. Before the occupational exposure, the mercuric urinary levels measured in several workers were below $3 \mathrm{ug} / \mathrm{g} \mathrm{Cr}$. However, no quantitative reference data were available about the level of mercury exposure at the time of the acute event.

Despite the range of early-stage symptoms, only three workers underwent early chelation with dimercaprol, also called British antiLewisite (BAL), after the initial exposure, which was interrupted prematurely by severe adverse reactions related to this compound.

Between September 2013 and the end of 2014, 44 of the 49 affected patients presented to the Clinical Toxicology Unit of the Medical Science Institute of the University of Valladolid, Valladolid, Spain, for independent assessments. After evaluation, different ancillary tests and actions were proposed based on each patient's clinical data. Likewise, belated chelation (around 8 to 12 months after the poisoning event) with oral 2,3-dimercapto-1-propanesulfonic acid was proposed for at least 1 week; it was chosen based on the severity criteria in their local hospitals and used with only 15 patients. Twenty-nine of 44 subjects who presented with any visual, neurologic, and/or systemic symptoms at the second evaluation were referred for a complete ophthalmologic evaluation at the Institute of Applied Ophthalmobiology (IOBA) Eye Institute of the University of Valladolid. Those without visual symptoms either at this time or in previous medical examinations were not considered for this evaluation.

\section{Ophthalmic Examination}

At the beginning of the study, careful case histories performed to rule out previous ocular, neural, or systemic diseases that could have affected the visual examinations.

Twenty-nine patients underwent a full ophthalmic examination that included measurement of intraocular pressure and best-corrected visual acuity (BCVA) using the Early Treatment Diabetic Retinopathy Scale (ETDRS), slit-lamp examination, funduscopy, and OCT, with particular attention to evaluation of the central retinal thickness (CRT) (3D-OCT 2000, Topcon Inc., Tokyo, Japan) and examination of the retinal nerve fiber layer thickness (RNFLT) (OCT Stratus 3000 Zeiss Meditec, Oberkochen, Germany). Color vision using the Roth 28 Hue Test (Lunean Ophtalmologie, Paris, France) and contrast sensitivity (CS) using the CSV-1000 chart (Vectorvision, Greenville, OH) were measured for all patients. The results of the Roth 28 Hue Test were scored in two ways. First, a color confusion index (CCI) was calculated for each participant for statistical analysis [11-13].

Second, a clinical diagnosis of the type of loss was determined by plotting the response on a standard score sheet. This allowed determination of the axis of color confusion. Based on the major confusion axis, a diagnosis of normal, red-green, blue-yellow, mixed, or non-specific deficiency was established.

The visual fields (VFs) were assessed using the Humphrey 750i Visual Field Analyzer (Carl Zeiss, Oberkochen, Germany) and the central $30-2$ SITA fast strategy protocol. Only tests that met the criteria [low $8<20 \%$ ), false positive, false negative, and fixation loss parameters] were evaluated. 
Pattern reversal visual-evoked potentials (PRVEP) and ERG recordings were assessed using a computerized Optoelectronic Stimulator Vision Monitor MonPack 120 Metrovision (Pérenchies, France) according to the International Society for Clinical Electrophysiology of Vision (ISCEV) protocols [14, 15]. We recorded full-field ERGs (ffERGs), PERGs, and multifocal mfERGs from both eyes of each patient with scaled hexagons stimulating 61 zones. Four patterns of abnormal mfERG amplitude responses were assessed: paracentral loss, foveal loss, peripheral loss, and generalized loss [16]. The technical data used for the electrophysiologic tests are shown in Table 1.

Table 1

Technical Data Used for Electrophysiology Examination

\begin{tabular}{|c|c|c|c|c|c|}
\hline Examination & $\begin{array}{l}\text { High pass Cut-off } \\
(\mathrm{Hz})\end{array}$ & $\begin{array}{l}\text { Low pass Cut-off } \\
(\mathrm{Hz})\end{array}$ & $\begin{array}{l}\text { Band Reject } \\
\text { Filter }\end{array}$ & $\begin{array}{l}\text { Amplification } \\
\text { Gain }\end{array}$ & $\begin{array}{l}\text { Impedance Threshold } \\
\text { (Kohms) }\end{array}$ \\
\hline SSR & 1 & 105 & 50 & 3125 & 10 \\
\hline MSR & 1 & 105 & 50 & 3125 & 10 \\
\hline OP & 100 & 288 & 50 & 3125 & 10 \\
\hline $\begin{array}{l}\text { Flicker } \\
30 \mathrm{~Hz}\end{array}$ & 10 & 96 & 50 & 3125 & 10 \\
\hline SFCR & 1 & 38 & 50 & 3125 & 10 \\
\hline PERG & 1 & 100 & off & 12.500 & 95 \\
\hline mERG & 0,1 & 72 & 50 & 100.000 & 4 \\
\hline PRVEP & 1 & 35 & 50 & 12.500 & 95 \\
\hline
\end{tabular}

\section{Additional Tests}

Nerve conductance was assessed by electromyography (EMG) to evaluate peripheral neuropathy using standard protocol using a computerized system (Nihon Kodhen, Model MEB-9400, Irvine, CA). Sensory and motor nerve conduction velocities were determined in the median and peroneal nerves. Amplitude $(\mu \mathrm{V})$, latency $(\mathrm{m} / \mathrm{s})$, and conductance $(\mathrm{m} / \mathrm{s})$ were evaluated.

\section{Statistical Analysis}

Statistical analyses were performed using SPSS statistics 17.0 (SPSS, Inc, Chicago, IL). The BCVA was recorded using the ETDRS scale and converted to the logarithm of minimal angle of resolution (logMAR) for statistical analysis. All VA results are expressed in logMAR units with Snellen equivalent in parenthesis. Categorical variables were analyzed using Fisher's exact test or chi-square test. The t-test was used to compare the mean values of the parametric values. Pearson's correlation test was used to evaluate the correlation between ophthalmic findings and mercury levels in the blood and urine. For data without normal distribution, continuous variables were analyzed using the Wilcoxon rank-sum test. For repeated measures, the Wilcoxon signed-rank test was used, and Spearman test was performed for the correlation non-normally distributed data. For all tests, $P<0.05$ was considered significant.

For statistical analyses, participants were divided in two groups: group $1(\mathrm{G} 1)(\mathrm{n}=14)$ was comprised of workers who did not undergo belated chelation treatment and group $2(G 2)(n=15)$ included those who underwent chelation after 8 to 12 months after the mercury poisoning. In addition, for the electrophysiology function assessment, we included two additional subgroups. Another subgroup (G3) included 11 of 29 patients who had the deeper and more extensive defects in the VF tests, i.e., concentric constriction and hemifield defect patterns. Finally, we included an age-matched healthy control group $(n=16)$.

\section{Results}

All patients were men (mean age, $40.62 \pm 8.05$, range, 25-56). The mean early-stage urinary mercury concentration after the event among all patients was $302.86 \pm 405.36 \mu \mathrm{g} / \mathrm{g}$ Cr; range, $10-1,830$ ); the mean blood mercury concentration was $392.93 \pm 273.85 \mu \mathrm{g} / \mathrm{L}$ (range, 26-961). Both measurements were increased significantly in G2 compared to those in G1. The control group included 16 agematched (age, $43.44 \pm 8.30$ years, $P=0.271$ ) healthy individuals with normal ophthalmologic evaluations. The main clinical baseline characteristics and the EMG results from the patients are summarized in Table 2. 
Table 2

Baseline Characteristics, Laboratory and Electromyography Findings

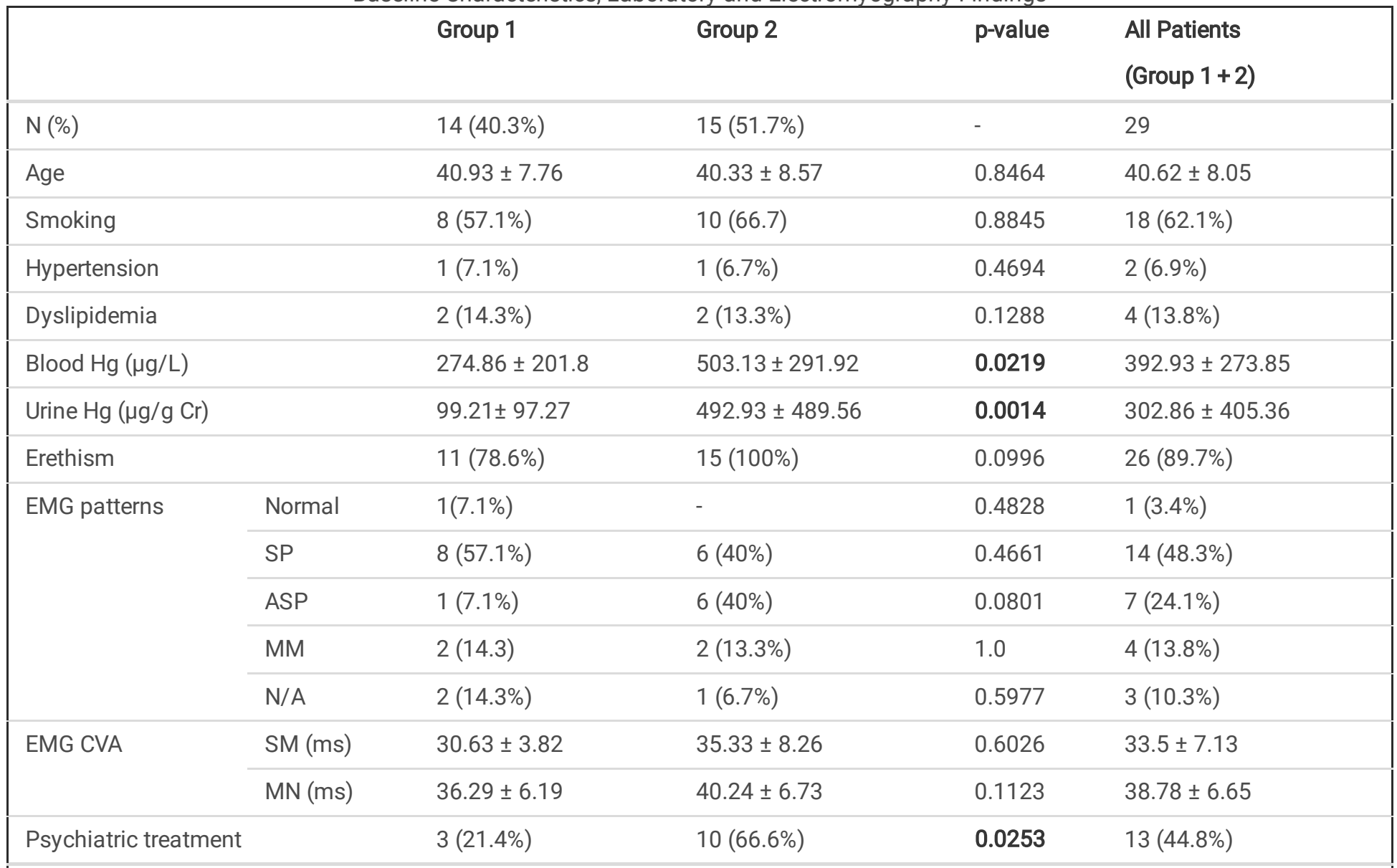

Group 1 = no chelation; Group 2 = late chelation; $\mathrm{EMG}=$ electromyography; $\mathrm{N}=$ number; $\mu \mathrm{g} / \mathrm{L}=$ microgram $/ \mathrm{liter} ; \mu \mathrm{g} / \mathrm{g} \mathrm{Cr}=\mathrm{microgram}$ per gram of creatinine; $\mathrm{SP}=$ sensorimotor polyneuropathy; $\mathrm{ASP}=$ axonal sensory polyneuropathy; $\mathrm{MM}=$ multiple mononeuropathy; $\mathrm{N} / \mathrm{A}=$ not performed. EMG CVA = Electromyography = conduction velocity assessment; $\mathrm{SN}=$ Sensory nerve; MN = Motor nerve; $\mathrm{ms}=$ milliseconds.

Reference values $[33,34]=$ normal velocity conduction in $\mathrm{SN}>40$ milliseconds. Normal velocity conduction in MN $>49$ milliseconds.

\section{Ophthalmologic Findings}

The main ophthalmic findings are shown in Table 3. The VA decreased $(<20 / 20)$ in nine $(64.3 \%)$ patients in G1 and in five $(33.3 \%)$ patients in G2, a difference that did not reach significance (Table 3). Fifteen (51.7\%) of 29 patients presented with additional unspecific ocular complaints such as dry eye or eye redness and light sensitivity. Table 3 and Fig. 1 show the VA, color vision, CS, VF, and OCT results. 
Table 3

Ophthalmic Examination Findings

\begin{tabular}{|c|c|c|c|c|c|c|c|c|c|c|}
\hline & & \multicolumn{2}{|l|}{ Group 1} & \multicolumn{2}{|l|}{ Group 2} & \multicolumn{2}{|l|}{$P$ Value } & \multicolumn{2}{|c|}{$\begin{array}{l}\text { All patients } \\
\text { (Group } 1+2)\end{array}$} & $\begin{array}{l}\text { Reference } \\
\text { Values }\end{array}$ \\
\hline \multicolumn{2}{|l|}{ N (eyes) } & \multicolumn{2}{|c|}{14 (28 eyes) } & \multicolumn{2}{|c|}{15 (30eyes) } & \multicolumn{2}{|l|}{-} & \multicolumn{2}{|c|}{29 (58eyes) } & - \\
\hline \multirow{2}{*}{\multicolumn{2}{|c|}{$\begin{array}{l}\text { BCVA Logmar } \\
\text { [Snellen] }\end{array}$}} & \multirow{2}{*}{\multicolumn{2}{|c|}{$\begin{array}{l}0.017 \pm 0.151 \\
{[0.887 \pm 0.238]}\end{array}$}} & \multicolumn{2}{|c|}{$0.078 \pm 0.865$} & \multicolumn{2}{|l|}{0.631} & \multicolumn{2}{|c|}{$0.048 \pm 0.126$} & $0.0[6 / 6]$ \\
\hline & & & & \multicolumn{2}{|c|}{$[0.950 \pm 0.172]$} & & & \multicolumn{2}{|c|}{$[0.920 \pm 0.205]$} & \\
\hline \multicolumn{2}{|l|}{ CVS } & \multicolumn{2}{|l|}{$7(50 \%)$} & \multicolumn{2}{|l|}{$6(40 \%)$} & \multirow{2}{*}{\multicolumn{2}{|c|}{0.715}} & \multicolumn{2}{|c|}{$13(44.8 \%)$} & - \\
\hline \multirow[t]{5}{*}{ Patterns } & Normal & \multicolumn{2}{|l|}{$7(50 \%)$} & \multicolumn{2}{|l|}{$9(60 \%)$} & & & \multicolumn{2}{|c|}{$16(55.2 \%)$} & - \\
\hline & Red-green defect & \multicolumn{2}{|l|}{1} & \multicolumn{2}{|l|}{1} & \multicolumn{2}{|l|}{1.0} & \multicolumn{2}{|c|}{$2(6.8 \%)$} & - \\
\hline & $\begin{array}{l}\text { Blue-yellow } \\
\text { defect }\end{array}$ & \multicolumn{2}{|l|}{5} & \multicolumn{2}{|l|}{4} & \multicolumn{2}{|l|}{0.699} & $9(31.03$ & & - \\
\hline & Mixed & 0 & & 1 & & 0.4828 & & $1(3.44 \%$ & & - \\
\hline & $\begin{array}{l}\text { Non-specific } \\
\text { deficiency }\end{array}$ & 1 & & 0 & & 1.0 & & $1(3.44 \%$ & & - \\
\hline $\mathrm{CCl}$ & & $1.872 \pm 1$ & 07 & $1.278 \pm 0$ & 97 & 0.146 & & $1.642 \pm$ & 183 & 1.0 \\
\hline CSA & Eye & $\mathrm{RE}$ & LE & $\mathrm{RE}$ & LE & $\mathrm{RE}$ & LE & $\mathrm{RE}$ & LE & - \\
\hline & CS3 & $\begin{array}{l}5.92 \pm \\
0.91\end{array}$ & $\begin{array}{l}5.50 \pm \\
1.12\end{array}$ & $\begin{array}{l}5.93 \pm \\
1.48\end{array}$ & $\begin{array}{l}5.86 \pm \\
1.12\end{array}$ & 0.9983 & 0.3878 & $\begin{array}{l}5.93 \pm \\
1.22\end{array}$ & $\begin{array}{l}5.69 \pm \\
1.16\end{array}$ & - \\
\hline & CS6 & $\begin{array}{l}5.57 \pm \\
0.85\end{array}$ & $\begin{array}{l}5.42 \pm \\
1.28\end{array}$ & $\begin{array}{l}5.66 \pm \\
1.29\end{array}$ & $\begin{array}{l}5.93 \pm \\
1.33\end{array}$ & 0.8295 & 0.3090 & $\begin{array}{l}5.62 \pm \\
1.08\end{array}$ & $\begin{array}{l}5.69 \pm \\
1.31\end{array}$ & - \\
\hline & CS12 & $\begin{array}{l}3.28 \pm \\
0.99\end{array}$ & $\begin{array}{l}3.42 \pm \\
1.01\end{array}$ & $\begin{array}{l}3.46 \pm \\
1.06\end{array}$ & $\begin{array}{l}3.53 \pm \\
0.83\end{array}$ & 0.6389 & 0.7647 & $\begin{array}{l}3.37 \pm \\
1.01\end{array}$ & $\begin{array}{l}3.48 \pm \\
0.91\end{array}$ & - \\
\hline & CS18 & $\begin{array}{l}3.35 \pm \\
1.27\end{array}$ & $\begin{array}{l}3.50 \pm \\
1.69\end{array}$ & $\begin{array}{l}3.33 \pm \\
1.44\end{array}$ & $\begin{array}{l}3.33 \pm \\
1.39\end{array}$ & 0.9577 & 0.7700 & $\begin{array}{l}3.34 \pm \\
1.34\end{array}$ & $\begin{array}{l}3.41 \pm \\
1.52\end{array}$ & - \\
\hline VF & Eye & $\mathrm{RE}$ & LE & $\mathrm{RE}$ & LE & $\mathrm{RE}$ & LE & $\mathrm{RE}$ & LE & - \\
\hline & MD & $\begin{array}{l}-5.52 \pm \\
7.33\end{array}$ & $\begin{array}{l}-6.88 \pm \\
8.18\end{array}$ & $\begin{array}{l}-5.76 \pm \\
8.7\end{array}$ & $\begin{array}{l}-6.86 \pm \\
9.11\end{array}$ & 0.8614 & 0.8272 & $\begin{array}{l}-5.64 \pm \\
7.92\end{array}$ & $\begin{array}{l}-6.87 \pm \\
8.52\end{array}$ & 0.0 \\
\hline & VFI & $\begin{array}{l}88.0 \pm \\
18.4\end{array}$ & $\begin{array}{l}85 \pm \\
20.3\end{array}$ & $\begin{array}{l}85.4 \pm \\
24.1\end{array}$ & $\begin{array}{l}85.7 \pm \\
23.0\end{array}$ & 0.7093 & 0.4294 & $\begin{array}{l}86.7 \pm \\
21.2\end{array}$ & $\begin{array}{l}85.4 \pm \\
21.4\end{array}$ & $100 \%$ \\
\hline & Total & $8(57.1 \%)$ & & $10(66.6$ & & 0.7104 & & $18(62$. & & - \\
\hline OCT & Eye & $\mathrm{RE}$ & LE & $\mathrm{RE}$ & LE & RE & LE & $\mathrm{RE}$ & LE & - \\
\hline & CRT & $\begin{array}{l}249.8 \pm \\
15.9\end{array}$ & $\begin{array}{l}248.9 \pm \\
18,8\end{array}$ & $\begin{array}{l}249.0 \pm \\
25.5\end{array}$ & $\begin{array}{l}247.4 \pm \\
23,5\end{array}$ & 0.9288 & 0.8537 & $\begin{array}{l}249.4 \\
\pm 21,0\end{array}$ & $\begin{array}{l}248.1 \\
\pm 20,7\end{array}$ & $\begin{array}{l}233.6 \pm \\
19.7\end{array}$ \\
\hline & RNFLT & $\begin{array}{l}103.2 \pm \\
13.2\end{array}$ & $\begin{array}{l}100.4 \pm \\
12.0\end{array}$ & $\begin{array}{l}101.3 \pm \\
7.77\end{array}$ & $\begin{array}{l}100.1 \pm \\
11.1\end{array}$ & 0.3536 & 0.3536 & $\begin{array}{r}102.2 \\
\pm 10.5\end{array}$ & $\begin{array}{r}100.2 \\
\pm 11.3\end{array}$ & $100 \pm 18$ \\
\hline $\begin{array}{l}\text { Group } 1( \\
\text { Electromy } \\
\text { CS3 = spa } \\
\text { CS18 = sp } \\
\text { coherence }\end{array}$ & $\begin{array}{l}\text { 1) = no chelation. G } \\
\text { graphy; CVS = colo } \\
\text { ial frequency at } 3 \text { C } \\
\text { tial frequency at } 18 \\
\text { tomography; CRT = }\end{array}$ & $\begin{array}{l}\text { up } 2 \text { (G2) } \\
\text { vision sco } \\
\text { les/degre } \\
\text { cycles/des } \\
\text { entral retir }\end{array}$ & $\begin{array}{l}\text { late chel } \\
\text { s; CCI = cc } \\
\text { CS6 = sp } \\
\text { ee; VF = vi } \\
\text { I thicknes }\end{array}$ & $\begin{array}{l}\text { n. BCVA } \\
\text { confusic } \\
\text { l frequen } \\
\text { al field tes } \\
\text { RNFLT = r }\end{array}$ & $\begin{array}{l}\text { est-correc } \\
\text { index; CS } \\
\text { at } 6 \text { cycle } \\
\text { MD = mea } \\
\text { nal nerve }\end{array}$ & $\begin{array}{l}\text { d visual a } \\
\text { alteratior } \\
\text { degree; C } \\
\text { deviation; } \\
\text { er layer th }\end{array}$ & $\begin{array}{l}\text { uity; RE = } \\
\text { s in the ac } \\
12=\text { spat } \\
\text { VFI = Visu } \\
\text { ickness. }\end{array}$ & $\begin{array}{l}\text { ight eye; } \\
\text { iromatic } \\
\text { al freque } \\
\text { I Field In }\end{array}$ & $\begin{array}{l}E=\text { left ey } \\
\text { ontrast s } \\
\text { y at } 12 \text { c } \\
\text { ex; OCT }\end{array}$ & $\begin{array}{l}\text { EMG = } \\
\text { Isitivity; } \\
\text { cles/degree; } \\
\text { ptical }\end{array}$ \\
\hline
\end{tabular}

Acquired dyschromatopsia, especially in the blue-yellow range of the Roth 28 Hue Test, occurred in 13 (44.8\%) patients. The mean CCI was $1.642 \pm 1.183$ (normal value, 1.0 and higher values indicate worse hue discrimination [11, 12]. No significant difference in the $\mathrm{CCl}$ was seen between G1 and G2 (Table 3). 
Twenty-eight (96.5\%) patients showed changes in the achromatic CS in at least one of the four spatial frequencies, but mainly in the higher ones; the difference between G1 and G2 did not reach significance (Table 3, Fig. 1).

Twenty-one (72.4\%) patients had VF alterations. The most prevalent patterns were concentric constriction (17 eyes, $29.3 \%)$, scattered defects (6 eyes, 10.3\%), hemifield defects respecting the horizontal and vertical meridians (5 eyes, 8.6\%), nasal defects (4 eyes, 6.9\%), and arcuate defects (2 eyes, 3.4\%). There were no significant differences between G1 and G2 in patterns, mean deviation, or VF index (VFI) (Table 3).

The OCT results showed no significant differences when the CRT and RNFLT measurements were compared to values in the normative SD-OCT databases $[17,18]$ between $\mathrm{G} 1$ and $\mathrm{G} 2$ (Table 3).

Correlation analyses between the blood mercury levels (BML) and all variables showed only a significant negative correlation with the BCVA (Table 4).

Table 4

Correlation between BML and BCVA

\begin{tabular}{|c|c|c|c|c|}
\hline BML & & Group 1 & Group 2 & $\begin{array}{l}\text { All patients } \\
\text { (Group } 1+2)\end{array}$ \\
\hline \multirow[t]{6}{*}{ BCVA } & \multirow[t]{2}{*}{ RE } & $r=-0.56$ & $r=-0.34$ & $r=-0.36$ \\
\hline & & $p=0.038$ & $p=0.204$ & $p=0.049$ \\
\hline & \multirow[t]{2}{*}{ LE } & $r=-0.54$ & $r=-0.32$ & $r=-0.37$ \\
\hline & & $p=0.042$ & $p=0.245$ & $p=0.042$ \\
\hline & \multirow[t]{2}{*}{ Global } & $r=-0.54$ & $r=-0.30$ & $r=-0.36$ \\
\hline & & $p=0.042$ & $p=0.274$ & $p=0.048$ \\
\hline \multicolumn{5}{|c|}{ Group 1 = no chelation; Group 2 = late chelation. BML = blood mercury levels; } \\
\hline
\end{tabular}

\section{Electrophysiology Function Assessment}

The ffERGs were recorded in 28 patients and 16 controls. No significant differences were found between G1 and G2 (Table 5 ). The ERG amplitudes from all mercury-contaminated patients (G1 plus G2) did not differ from those of controls for the 30-Hz flicker and singleflash cone response (SFCR). The a- and b-wave amplitudes did not differ significantly between patients and controls in the scotopic rod response (SRR) and maximal scotopic response (MSR), respectively (Table 5). 
Table 5

Full-Field ERGs. Amplitude of a- and b-Waves for the SRR, MSR, OP, Flicker $30 \mathrm{~Hz}$ and SFCR

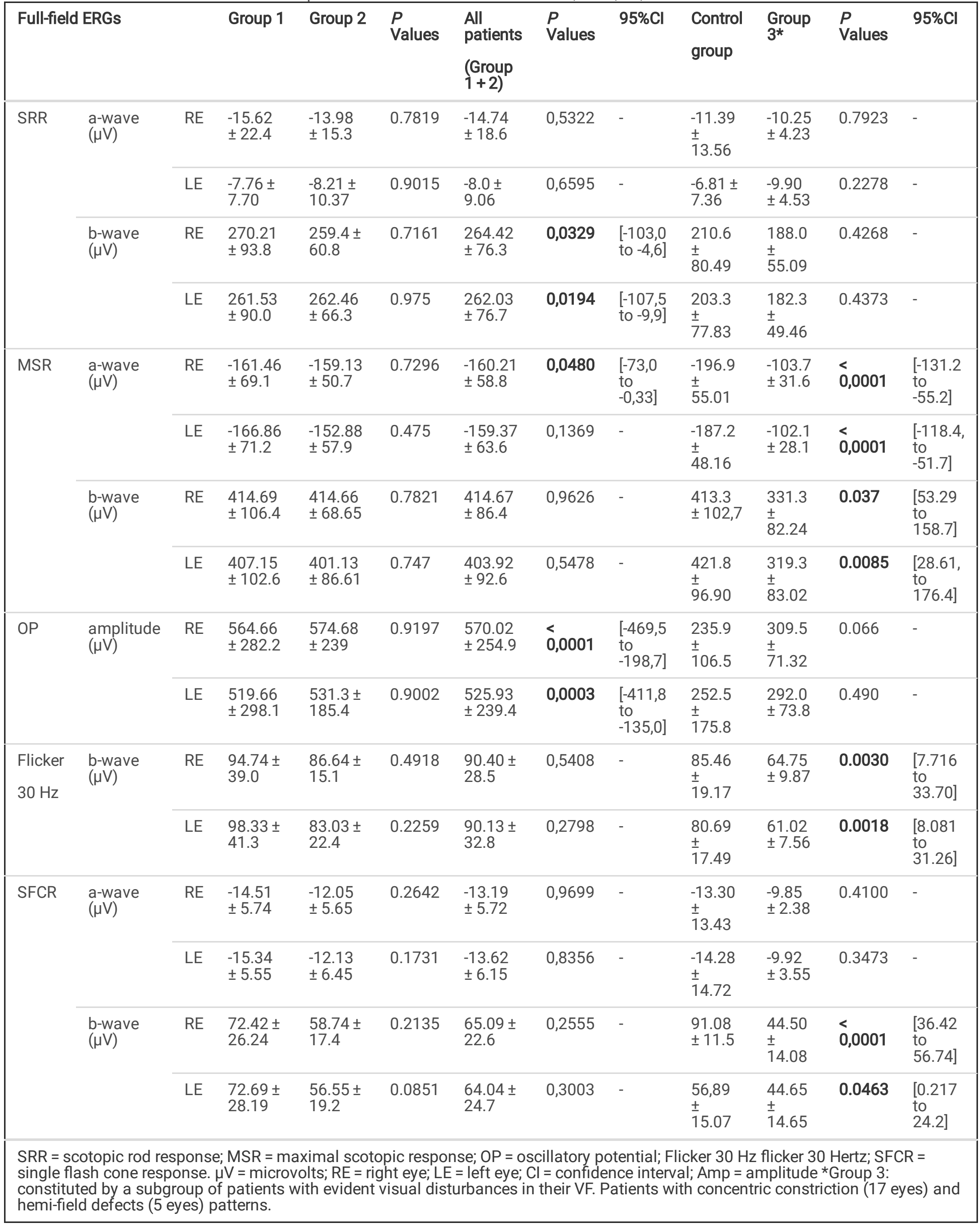


Statistical differences were found in all patients (G1 plus G2) and controls for the b-wave in SRR, a-wave in MSR, and the sum of oscillatory potential (OP) protocols (Table 5).

Because about half of the patients had no visual impairment, the electrophysiologic measurement averages from the global sample (G1 plus G2) could have been falsely near normal. Thus, we focused on the results obtained from patients with relevant impaired VF tests (G3) compared to the control group. These data showed lower amplitudes in subgroup G3 compared to the global sample in all ERG parameters reported previously [6]. These differences were significant for MSR and 30-Hz flicker for the a- and b-waves and for the bwave in the SFCR (Table 5).

A negative significant correlation was seen in $\mathrm{G} 1$ between the BML and ERG amplitudes of the b-wave in SRR, MSR, sum of the OP, and in 30- $\mathrm{Hz}$ flicker responses and sum of the OP in $\mathrm{G} 2$ (Table 6).

Table 6

Correlation BML and b-Wave Amplitudes for the SRR, MSR, OP, Flicker $30 \mathrm{~Hz}$ and SFCR

\begin{tabular}{|c|c|c|c|c|c|c|c|c|}
\hline \multirow{2}{*}{\multicolumn{2}{|c|}{$\begin{array}{l}\text { BML } \\
\text { Full-field ERGs }\end{array}$}} & \multirow{3}{*}{$\begin{array}{l}\text { Eye } \\
\text { RE }\end{array}$} & \multirow[t]{2}{*}{ Group 1} & & \multirow[t]{2}{*}{ Group 2} & & \multirow{2}{*}{\multicolumn{2}{|c|}{$\begin{array}{l}\text { All patients } \\
\text { (Group } 1+2)\end{array}$}} \\
\hline & & & & & & & & \\
\hline \multirow[t]{2}{*}{ SRR } & \multirow[t]{2}{*}{ b-wave $(\mu \mathrm{V})$} & & $r=0.49$ & $p=0.084$ & $r=-0.35$ & $p=0.197$ & $r=-0.02$ & $p=0.918$ \\
\hline & & LE & $r=0.61$ & $p=0.025$ & $r=-0.12$ & $p=0.648$ & $r=0.18$ & $p=0.353$ \\
\hline \multirow[t]{2}{*}{ MSR } & \multirow[t]{2}{*}{ b-wave $(\mu \mathrm{V})$} & $\mathrm{RE}$ & $r=0.61$ & $p=0.024$ & $r=-0.17$ & $p=0.528$ & $r=0.08$ & $p=0.918$ \\
\hline & & LE & $r=0.81$ & $p=0.001$ & $r=-0.02$ & $p=0.935$ & $r=0.26$ & $p=0.171$ \\
\hline \multirow[t]{2}{*}{ Flicker $30 \mathrm{~Hz}$} & \multirow[t]{2}{*}{ b-wave $(\mu \mathrm{V})$} & $\mathrm{RE}$ & $r=-0.06$ & $p=0.825$ & $r=-0.51$ & $p=0.047$ & $r=-0.24$ & $p=0.214$ \\
\hline & & LE & $r=0.06$ & $p=0.841$ & $r=0.08$ & $p=0.772$ & $r=-0.04$ & $p=0.812$ \\
\hline \multirow[t]{2}{*}{$\mathrm{OP}$} & \multirow[t]{2}{*}{ Amplitude $(\mu \mathrm{V})$} & $\mathrm{RE}$ & $r=0.06$ & $p=0.023$ & $r=-0.57$ & $p=0.024$ & $r=-0.05$ & $p=0.771$ \\
\hline & & LE & $r=0.70$ & $p=0.007$ & $r=-0.47$ & $p=0.073$ & $r=0.10$ & $p=0.607$ \\
\hline \multirow[t]{2}{*}{ SFCR } & \multirow[t]{2}{*}{ b-wave $(\mu \mathrm{V})$} & $\mathrm{RE}$ & $r=0.19$ & $p=0.531$ & $r=-0.31$ & $p=0.259$ & $r=-0.19$ & $p=0.327$ \\
\hline & & LE & $r=0.26$ & $p=0.380$ & $r=-0.07$ & $p=0.787$ & $r=0.07$ & $p=0.708$ \\
\hline
\end{tabular}

\section{PERG}

PERG was performed in 27 right eyes and in 26 left eyes of patients and in 14 controls (both eyes) (Table 7). Despite showing reduced amplitudes in P50 and N95, there were no significant differences between all patients (G1 plus G2) and the amplitudes obtained in the control group. 
Table 7

PERG in Patients and Control Group

\begin{tabular}{|c|c|c|c|c|c|c|c|c|c|c|c|}
\hline \multicolumn{3}{|c|}{ PERG } & Group 1 & Group 2 & $\begin{array}{l}P \\
\text { Values }\end{array}$ & $\begin{array}{l}\text { All } \\
\text { patients } \\
\text { (Group } 1 \\
+2 \text { ) }\end{array}$ & $\begin{array}{l}\text { Control } \\
\text { Values }\end{array}$ & $\begin{array}{l}P \\
\text { Values }\end{array}$ & $\begin{array}{l}\text { Group } \\
3^{*}\end{array}$ & $\begin{array}{l}P \\
\text { Values }\end{array}$ & $95 \% \mathrm{Cl}$ \\
\hline \multirow[t]{2}{*}{ P50 } & $\begin{array}{l}\text { Amplitude } \\
(\mu \mathrm{V})\end{array}$ & $\mathrm{RE}$ & $\begin{array}{l}4.2 \pm \\
1.59\end{array}$ & $\begin{array}{l}4.41 \pm \\
1.5\end{array}$ & 0.9805 & $\begin{array}{l}4.32 \pm \\
1.51\end{array}$ & $4.92 \pm 1.66$ & 0.2454 & $\begin{array}{l}3.13 \pm \\
0.67\end{array}$ & 0.0014 & $\begin{array}{l}{[0.76 \text { to }} \\
2.81]\end{array}$ \\
\hline & & LE & $\begin{array}{l}4.56 \pm \\
1.93\end{array}$ & $\begin{array}{l}4.19 \pm \\
1.72\end{array}$ & 0.6044 & $\begin{array}{l}4.35 \pm \\
1.78\end{array}$ & $4.58 \pm 0.91$ & 0.6052 & $\begin{array}{l}2.93 \pm \\
0.81\end{array}$ & 0.0232 & $\begin{array}{l}{[0.21 \text { to }} \\
2.61]\end{array}$ \\
\hline \multirow[t]{2}{*}{ N95 } & $\begin{array}{l}\text { Amplitude } \\
(\mu \mathrm{V})\end{array}$ & $\mathrm{RE}$ & $\begin{array}{l}-5.98 \\
\pm .548\end{array}$ & $\begin{array}{l}-5.84 \\
\pm .314\end{array}$ & 0.4719 & $\begin{array}{l}-5.90 \\
\pm .293\end{array}$ & $\begin{array}{l}-6.65 \pm \\
2.06\end{array}$ & 0.0680 & $\begin{array}{l}-4.00 \pm \\
0.84\end{array}$ & 0.0006 & $\begin{array}{l}{[-4.01 \text { to }} \\
1.27]\end{array}$ \\
\hline & & LE & $\begin{array}{l}-5.04 \\
\pm .688\end{array}$ & $\begin{array}{l}-5.30 \\
\pm .637\end{array}$ & 0.1126 & $\begin{array}{l}-5.19 \\
\pm .460\end{array}$ & $\begin{array}{l}-5.65 \pm \\
1.63\end{array}$ & 0.1759 & $\begin{array}{l}-3.19 \pm \\
0.94\end{array}$ & 0.0002 & $\begin{array}{l}{[-3.60 \text { to }} \\
1.31]\end{array}$ \\
\hline \multicolumn{12}{|c|}{$\begin{array}{l}\text { Amp = amplitude; } P E R G=\text { pattern electroretinogram; } C l=\text { confidence interval; } \mu \mathrm{V}=\text { microvolts; } R E=\text { right eye; LE = left eye; } \mathrm{G} 1=\mathrm{Group} \\
1 ; \mathrm{G} 2=\text { Group } 2 .\end{array}$} \\
\hline
\end{tabular}

However, this trend seen in all patients became significant when patients with impaired VFs (G3) were compared with the control group (Table 7), which agreed with previous studies [19].

No significant differences were seen when the implicit times in both P50 and N95 components were compared. There was no correlation between the PERG values and the BML.

\section{PRVEP}

PRVEP was recorded in 29 workers and 14 controls. The average implicit times of P100 and amplitudes did not differ between all patients and the control group for the 60- and 30-minute checkerboard stimuli except in the implicit times of P100 for both 60 and 30 for the left eyes (Table 8); but significant differences were seen when G3 was compared to the control group (Table 8). However, no significant differences were seen when G1 and G2 were compared (Table 8); and no correlation was seen between the PRVEP and BML. 
Table 8

PRVER in Patients and Control Group

\begin{tabular}{|c|c|c|c|c|c|c|c|c|c|c|c|c|}
\hline \multicolumn{3}{|l|}{ PRVEP } & \multirow{2}{*}{$\begin{array}{l}\text { Group } 1 \\
\\
\begin{array}{l}9.72 \pm \\
4.69\end{array}\end{array}$} & \multirow{2}{*}{$\begin{array}{l}\text { Group } \\
2 \\
7.97 \pm \\
3.94\end{array}$} & \multirow{2}{*}{$\begin{array}{l}\boldsymbol{P} \\
\text { Values } \\
0.2853\end{array}$} & \multirow{2}{*}{$\begin{array}{l}\begin{array}{l}\text { All } \\
\text { patients }\end{array} \\
\text { (Group } \\
1+2) \\
8.82 \pm \\
4.33\end{array}$} & \multirow{3}{*}{$\begin{array}{l}\text { Control } \\
\text { Values } \\
\\
7.29 \pm \\
1.59\end{array}$} & \multirow{2}{*}{$\begin{array}{l}\boldsymbol{P} \\
\text { Values } \\
0,2852\end{array}$} & \multirow{2}{*}{$\begin{array}{l}95 \% \\
\mathrm{Cl} \\
-\end{array}$} & \multirow{3}{*}{$\begin{array}{l}\text { Group } \\
3^{*} \\
\\
5.05 \\
\pm 1.03\end{array}$} & \multirow{3}{*}{$\begin{array}{l}P \\
\text { Values } \\
0.0011\end{array}$} & \multirow{3}{*}{$\begin{array}{l}95 \% \mathrm{Cl} \\
\\
{[1.020} \\
\text { to } \\
3.450]\end{array}$} \\
\hline $\begin{array}{l}\text { P100- } \\
\text { Da } 60\end{array}$ & $\begin{array}{l}\text { Amplitude } \\
(\mu \mathrm{V})\end{array}$ & $\mathrm{RE}$ & & & & & & & & & & \\
\hline \multirow[t]{3}{*}{ R Lob } & & LE & $\begin{array}{l}8.82 \pm \\
4.54\end{array}$ & $\begin{array}{l}8.11 \pm \\
4.32\end{array}$ & 0.6467 & $\begin{array}{l}8.45 \pm \\
4.36\end{array}$ & & 0,4193 & - & & & \\
\hline & $\begin{array}{l}\text { Latency } \\
\text { (ms) }\end{array}$ & $\mathrm{RE}$ & $\begin{array}{l}116.78 \\
\pm 13.24\end{array}$ & $\begin{array}{l}113.6 \\
\pm 6.39\end{array}$ & 0.4211 & $\begin{array}{l}115.17 \\
\pm 10.21\end{array}$ & \multirow[t]{2}{*}{$\begin{array}{l}112.6 \pm \\
7.19\end{array}$} & 0,4684 & - & \multirow[t]{2}{*}{$\begin{array}{l}130.9 \\
\pm 8.17\end{array}$} & \multirow[t]{2}{*}{$<.0001$} & \multirow{2}{*}{$\begin{array}{l}{[-25,36} \\
\text { to } \\
-11,24]\end{array}$} \\
\hline & & LE & $\begin{array}{l}119.4 \pm \\
11.93\end{array}$ & $\begin{array}{l}117.5 \\
\pm 7.51\end{array}$ & 0.5993 & $\begin{array}{l}118.06 \\
\pm 9.71\end{array}$ & & 0,1127 & - & & & \\
\hline $\begin{array}{l}\text { P100- } \\
\text { Da } 60\end{array}$ & $\begin{array}{l}\text { Amplitude } \\
(\mu \mathrm{V})\end{array}$ & RE & $\begin{array}{l}8.93 \pm \\
4.07\end{array}$ & $\begin{array}{l}8.83 \pm \\
3.67\end{array}$ & 0.9476 & $\begin{array}{l}8.88 \pm \\
7.44\end{array}$ & $\begin{array}{l}6.33 \pm \\
2.40\end{array}$ & 0,2974 & - & $\begin{array}{l}5.15 \\
\pm 1.02\end{array}$ & 0.1538 & - \\
\hline \multirow[t]{3}{*}{ L Lob } & & LE & $\begin{array}{l}8.41 \pm \\
3.36\end{array}$ & $\begin{array}{l}7.61 \pm \\
3.97\end{array}$ & 0.5608 & $8 \pm 3.65$ & & 0,1872 & - & & & \\
\hline & $\begin{array}{l}\text { Latency } \\
\text { (ms) }\end{array}$ & RE & $\begin{array}{l}114.64 \\
\pm 12.15\end{array}$ & $\begin{array}{l}113.4 \\
\pm 5.73\end{array}$ & 0.8264 & $\begin{array}{l}114 \pm \\
9.24\end{array}$ & \multirow[t]{2}{*}{$\begin{array}{l}111.2 \pm \\
4.80\end{array}$} & 0.3681 & - & \multirow[t]{2}{*}{$\begin{array}{l}132.0 \\
\pm 7.09\end{array}$} & \multirow[t]{2}{*}{$\begin{array}{l}<.0001 \\
\end{array}$} & \multirow{2}{*}{$\begin{array}{l}{[-26,39} \\
\text { to } \\
-15,21]\end{array}$} \\
\hline & & LE & $\begin{array}{l}119.4 \pm \\
12.31\end{array}$ & $\begin{array}{l}117.5 \\
\pm 8.49\end{array}$ & 0.6173 & $\begin{array}{l}118.47 \\
\pm 10.36\end{array}$ & & 0.0420 & $\begin{array}{l}{[-14,1} \\
\text { to } \\
-0,27]\end{array}$ & & & \\
\hline $\begin{array}{l}\text { P100- } \\
\text { Da } 30\end{array}$ & $\begin{array}{l}\text { Amplitude } \\
(\mu \mathrm{V})\end{array}$ & $\mathrm{RE}$ & $\begin{array}{l}8.1 \pm \\
4.3\end{array}$ & $\begin{array}{l}8.47 \pm \\
3.23\end{array}$ & 0.7926 & $\begin{array}{l}8.29 \pm \\
3.72\end{array}$ & $\begin{array}{l}6.29 \pm \\
2.16\end{array}$ & 0,1179 & - & $\begin{array}{l}4.40 \\
\pm 1.56\end{array}$ & 0,0319 & $\begin{array}{l}{[0,1813} \\
\text { to } 3,59]\end{array}$ \\
\hline \multirow[t]{3}{*}{ R Lob } & & LE & $\begin{array}{l}7.65 \pm \\
4.4\end{array}$ & $\begin{array}{l}7.24 \pm \\
3.53\end{array}$ & 0.7835 & $\begin{array}{l}7.44 \pm \\
3.91\end{array}$ & & 0,3846 & - & & & \\
\hline & $\begin{array}{l}\text { Latency } \\
\text { (ms) }\end{array}$ & $\mathrm{RE}$ & $\begin{array}{l}116.57 \\
\pm 6.81\end{array}$ & $\begin{array}{l}117 \pm \\
5.83\end{array}$ & 0.8566 & $\begin{array}{l}116.79 \\
\pm 6.21\end{array}$ & \multirow[t]{2}{*}{$\begin{array}{l}112.12 \\
\pm 8.02\end{array}$} & 0,0588 & - & \multirow[t]{2}{*}{$\begin{array}{l}134.7 \\
\pm 15.6\end{array}$} & \multirow[t]{2}{*}{0,0006} & \multirow{2}{*}{$\begin{array}{l}{[-34,03} \\
\text { to } \\
-10,97]\end{array}$} \\
\hline & & LE & $\begin{array}{l}124.43 \\
\pm 18.42\end{array}$ & $\begin{array}{l}120.47 \\
\pm 6.48\end{array}$ & 0.9474 & $\begin{array}{l}122.38 \\
\pm 13.51\end{array}$ & & 0,0283 & $\begin{array}{l}{[-19,5} \\
\text { to } \\
-1,16]\end{array}$ & & & \\
\hline $\begin{array}{l}\text { P100- } \\
\text { Da } 30\end{array}$ & $\begin{array}{l}\text { Amplitude } \\
(\mu \mathrm{V})\end{array}$ & $\mathrm{RE}$ & $\begin{array}{l}8.2 \pm \\
4.56\end{array}$ & $\begin{array}{l}7.71 \pm \\
3.56\end{array}$ & 0.7471 & $\begin{array}{l}7.94 \pm \\
4.01\end{array}$ & $\begin{array}{l}5.93 \pm \\
2.01\end{array}$ & 0,1392 & - & $\begin{array}{l}3.85 \\
\pm 1.44\end{array}$ & 0,0128 & $\begin{array}{l}{[0,494} \\
\text { to } 3,66]\end{array}$ \\
\hline \multirow[t]{3}{*}{ L Lob } & & LE & $\begin{array}{l}6.85 \pm \\
4.74\end{array}$ & $\begin{array}{l}6.14 \pm \\
2.73\end{array}$ & 0.6222 & $\begin{array}{l}6.48 \pm \\
3.78\end{array}$ & & 0,6649 & - & & & \\
\hline & $\begin{array}{l}\text { Latency } \\
\text { (ms) }\end{array}$ & $\mathrm{RE}$ & $\begin{array}{l}116 \pm \\
8.03\end{array}$ & $\begin{array}{l}118.2 \\
\pm 6.97\end{array}$ & 0.4367 & $\begin{array}{l}117.14 \\
\pm 7.45\end{array}$ & \multirow[t]{2}{*}{$\begin{array}{l}112.4 \pm \\
7.32\end{array}$} & 0,0898 & - & \multirow[t]{2}{*}{$\begin{array}{l}136.8 \\
\pm 22.4\end{array}$} & \multirow[t]{2}{*}{0,0039} & \multirow{2}{*}{$\begin{array}{l}{[-39,96} \\
\text { to } \\
-8,84]\end{array}$} \\
\hline & & LE & $\begin{array}{l}126.14 \\
\pm 24.24\end{array}$ & $\begin{array}{l}119.93 \\
\pm 6.13\end{array}$ & 0.5524 & $\begin{array}{l}122.93 \\
\pm 17.36\end{array}$ & & 0,0724 & - & & & \\
\hline \multicolumn{13}{|c|}{$\begin{array}{l}\text { PRVF = pattern reversal visual evoked potential; P100-Da } 60=\text { P100 wave with } 60 \text { ' checkerboard stimuli; P100-Da } 30 \text { = P100 wave } \\
\text { with } 30 \text { ' checkerboard stimuli; R Lob = right occipital cortex; L Lob = left occipital cortex; } \mu \mathrm{V}=\text { microvolts; RE = right eye; } \mathrm{LE}=\text { left eye; } \\
\mathrm{Cl}=\text { confidence interval. }\end{array}$} \\
\hline
\end{tabular}

\section{MfERG}

The mfERGs were recorded in 26 of 29 patients and 11 controls. The most prevalent patterns were peripheral loss ( 16 eyes, $30.7 \%)$ and central loss (8 eyes, $15.4 \%$ ), followed by paracentral defects ( 6 eyes, $11.5 \%$ ). Conversely, no depressed amplitude responses to the ffERG were observed in 22 eyes (42.3\%). Because the peripheral pattern was the most frequently found, the N1/P1 amplitude ratio in the peripheral rings of the mfERG was analyzed; a significantly lower value was seen in all patients (G1 plus G2) in rings $5^{\circ}$ to $10^{\circ}$ and $>15^{\circ}$ compared to the control group. The data showed additional significant differences at rings $1,<2^{\circ}$, ring $2,2^{\circ}$ to $5^{\circ}$, ring $3,5^{\circ}$ to $10^{\circ}$, ring 5 , 
and $>15^{\circ}$ when $\mathrm{G} 3$ was compared to controls. These results agreed with previous studies $[16,20]$. No significant differences were seen when the mfERG patterns in G1 and G2 were compared (Table 9) and no correlations were seen between the mfERG values at rings 2$5^{\circ}, 5^{\circ}-10^{\circ}, 10^{\circ}$ to $15^{\circ}$, and $>15^{\circ}$ and the BML.

Table 9

mfERG Values in Patients and Control Group

\begin{tabular}{|c|c|c|c|c|c|c|c|c|c|c|c|c|}
\hline \multirow[t]{11}{*}{$\begin{array}{l}\text { Amplitude } \\
\text { P1/N1ratio }\end{array}$} & Ring & Eye & $\begin{array}{l}\text { Group } \\
1\end{array}$ & $\begin{array}{l}\text { Group } \\
2\end{array}$ & $\begin{array}{l}P \\
\text { Value }\end{array}$ & $\begin{array}{l}\text { All } \\
\text { patients } \\
\text { (Group } \\
1+2)\end{array}$ & $\begin{array}{l}\text { Control } \\
\text { Values }\end{array}$ & $\begin{array}{l}P \\
\text { Value }\end{array}$ & $95 \% \mathrm{Cl}$ & $\begin{array}{l}\text { Group } \\
3^{*}\end{array}$ & $\begin{array}{l}P \\
\text { Value }\end{array}$ & $95 \% \mathrm{Cl}$ \\
\hline & $\begin{array}{l}\text { Ring } \\
1\end{array}$ & RE & $\begin{array}{l}378.3 \\
\pm 202.3\end{array}$ & $\begin{array}{l}438.8 \\
\pm 178.3\end{array}$ & 0,5050 & $\begin{array}{l}380 \pm \\
209.6\end{array}$ & $\begin{array}{l}528 \pm \\
104,7\end{array}$ & 0,0334 & $\begin{array}{l}{[12,35} \\
\text { to } \\
283,7]\end{array}$ & $\begin{array}{l}298.6 \\
\pm \\
137.4\end{array}$ & 0.0003 & $\begin{array}{l}{[120,8} \\
\text { to } \\
338,0]\end{array}$ \\
\hline & & LE & $\begin{array}{l}249 \pm \\
121.5\end{array}$ & $\begin{array}{l}303.4 \\
\pm 72.76\end{array}$ & 0,1731 & $\begin{array}{l}278.4 \pm \\
100.1\end{array}$ & & $\begin{array}{l}< \\
0,0001\end{array}$ & $\begin{array}{l}{[175,5} \\
\text { to } \\
323,7]\end{array}$ & $\begin{array}{l}182.0 \\
\pm \\
64.13\end{array}$ & $\begin{array}{l}< \\
0,0001\end{array}$ & $\begin{array}{l}{[268,8} \\
\text { to } \\
423,2]\end{array}$ \\
\hline & $\begin{array}{l}\text { Ring } \\
2\end{array}$ & RE & $\begin{array}{l}108,5 \\
\pm 46,23\end{array}$ & $\begin{array}{l}137,2 \pm \\
27,44\end{array}$ & 0,0623 & $\begin{array}{l}124 \pm \\
39.30\end{array}$ & $\begin{array}{l}122.8 \pm \\
12.69\end{array}$ & 0,9222 & - & $\begin{array}{l}89.99 \\
\pm \\
29.32\end{array}$ & 0.0028 & $\begin{array}{l}{[12,72} \\
\text { to } \\
52,90]\end{array}$ \\
\hline & & LE & $\begin{array}{l}109,5 \\
\pm 50,09\end{array}$ & $\begin{array}{l}134,9 \pm \\
32,34\end{array}$ & 0,1328 & $\begin{array}{l}123.2 \pm \\
42.59\end{array}$ & & 0,9760 & - & $\begin{array}{l}81.45 \\
\pm \\
27.35\end{array}$ & 0.0002 & $\begin{array}{l}{[22,39} \\
\text { to } \\
60,31]\end{array}$ \\
\hline & $\begin{array}{l}\text { Ring } \\
3\end{array}$ & RE & $\begin{array}{l}45.30 \\
\pm 8.96\end{array}$ & $\begin{array}{l}38.92 \\
\pm 12.4\end{array}$ & 0.1415 & $\begin{array}{l}42.35 \pm \\
10.95\end{array}$ & $\begin{array}{l}56.47 \pm \\
6.81\end{array}$ & 0,3031 & - & $\begin{array}{l}32.55 \\
\pm \\
7.470\end{array}$ & $\begin{array}{l}< \\
0,0001\end{array}$ & $\begin{array}{l}{[18,56} \\
\text { to } \\
31,28]\end{array}$ \\
\hline & & LE & $\begin{array}{l}45.8 \pm \\
7.22\end{array}$ & $\begin{array}{l}41.2 \pm \\
12.58\end{array}$ & 0.2596 & $\begin{array}{l}43.74 \pm \\
10.10\end{array}$ & & 0,3503 & - & $\begin{array}{l}34.43 \\
\pm \\
6.273\end{array}$ & $\stackrel{<}{0,0001}$ & $\begin{array}{l}{[17,21} \\
\text { to } \\
28,87]\end{array}$ \\
\hline & $\begin{array}{l}\text { Ring } \\
4\end{array}$ & RE & $\begin{array}{l}46.28 \\
\pm 10.06\end{array}$ & $\begin{array}{l}39.2 \pm \\
14.89\end{array}$ & 0.163 & $\begin{array}{l}43.01 \pm \\
12.77\end{array}$ & $\begin{array}{l}35.85 \pm \\
4.72\end{array}$ & 0,4736 & - & $\begin{array}{l}31.11 \\
\pm \\
8.031\end{array}$ & 0.1070 & - \\
\hline & & LE & $\begin{array}{l}46.7 \pm \\
6.95\end{array}$ & $\begin{array}{l}38.7 \pm \\
14.59\end{array}$ & 0.1728 & $\begin{array}{l}43.06 \pm \\
11.62\end{array}$ & & 0,4646 & - & $\begin{array}{l}32.08 \\
\pm \\
8.246\end{array}$ & 0.2031 & - \\
\hline & $\begin{array}{l}\text { Ring } \\
5\end{array}$ & RE & $\begin{array}{l}12.9 \pm \\
2.48\end{array}$ & $\begin{array}{l}11.7 \pm \\
3.89\end{array}$ & 0.3513 & $\begin{array}{l}12.36 \pm \\
3.20\end{array}$ & $\begin{array}{l}19.76 \pm \\
1.21\end{array}$ & $\stackrel{<}{0,0001}$ & $\begin{array}{l}{[5,370} \\
\text { to } \\
9,430]\end{array}$ & $\begin{array}{l}9.479 \\
\pm \\
1.489\end{array}$ & $\begin{array}{l}< \\
0,0001\end{array}$ & $\begin{array}{l}{[9,074} \\
\text { to } \\
11,49]\end{array}$ \\
\hline & & LE & $\begin{array}{l}13.2 \pm \\
2.0\end{array}$ & $\begin{array}{l}11.7 \pm \\
3.82\end{array}$ & 0.2608 & $\begin{array}{l}12.5 \pm \\
3.0\end{array}$ & & $\begin{array}{l}< \\
0,0001\end{array}$ & $\begin{array}{l}{[5,349} \\
\text { to } \\
9,171]\end{array}$ & $\begin{array}{l}9.802 \\
\pm \\
1.929\end{array}$ & $\begin{array}{l}<, 0001 \\
0,0\end{array}$ & $\begin{array}{l}{[8,526} \\
\text { to } \\
11,39]\end{array}$ \\
\hline
\end{tabular}

Amplitude P1/N1 ratio $\left(\mathrm{nV} / \mathrm{deg}^{2}\right)$.

Ring $1=<2^{\circ}$; ring $2=2-5^{\circ}$; ring $3=5^{\circ}-10^{\circ}$; ring $4=10^{\circ}-15^{\circ}$; and ring $5=>15^{\circ} ; \mathrm{RE}=$ right eye; $\mathrm{LE}=$ left eye; $\mathrm{Cl}=$ confidence interval.

*Group 3 is a subgroup of patients with evident visual disturbances in their visual field test. Patients with concentric constriction (17 eyes) and hemi-field defects (5 eyes) patterns.

Although comparability between the mfERG and perimetry test was limited, we assessed the VF defects patterns with the mfERG dysfunction patterns obtained in the three-dimensional plots. With limitations, we subjectively assessed these patterns based on the comparative methods and approaches used previously [21, 22]. The data showed that 14 (48.3\%) patients showed different patterns between the mfERG defects and the total deviation of visual sensitivities less than $5 \%$ recorded in their VF tests. Eight (27.5\%) patients had similar peripheral pattern defects in both tests, and four (13.8\%) patients had mixed patterns; in three (10.3\%) we could not establish any comparison. No differences were seen between groups G1 and G2. 
EMG, performed in 27 of 29 workers, showed different abnormality patterns and decreased nerve conduction velocity in most patients (Table 2). There were no significant differences between $\mathrm{G} 1$ and G2. No correlation was seen between the BML, nerve conduction velocity, and the P100 component in PRVEP in the entire sample.

\section{Discussion}

This study focused especially on retinal and visual function examinations because of the possibility of evaluating patients using new OCT and ERG techniques.

Mercury vapor is a significant source of mercuric load in occupational exposure because it is odorless and colorless and tends to accumulate in poorly ventilated areas. Once the lungs have absorbed the inhaled vapor, the mercury can reach different tissues via the bloodstream, with the primary target the CNS and eyes because of proximity $[2,23]$. When it is oxidized, it cannot penetrate the bloodbarrier again and remains for prolonged periods in tissues $[2,7,8,23]$.

As mentioned, the neurologic and thus the visual pathway effects resulting from mercury toxicity have been described widely $[2,23$, 24].The long-term exposure effects can include symptoms from tremor, neuropathy, personality changes referred to as mercurial erethism, speech disruption, delirium or rigidity to symptoms of VF defects, reduced VA, color and night vision, or decreased CS [2, 23, 25, 26]. However, the introduction of electrophysiology testing has established the presence of primary retinal involvement and that not all alterations of the visual pathway are due to CNS poisoning [6].

As mentioned, the first patient complaints were attributed to a viral infection, which delayed the diagnosis. Thus, when diagnosed correctly, the mercuric values in urine (mean, $302.86 \mu \mathrm{g} / \mathrm{g} \mathrm{Cr}$ ) and blood (mean, $392.93 \mu \mathrm{g} / \mathrm{L}$ ) significantly exceeded the maximal accepted level for occupational exposure $(<30 \mu \mathrm{g} / \mathrm{g}$ Cr and $10 \mu \mathrm{g} / \mathrm{L}$, respectively) $[9,10]$. In such cases, the mainstay of treatment is chelation therapy; however, only three patients underwent early chelation, which was stopped prematurely because of severe adverse reactions. Fifteen workers underwent delayed chelation ( 8 to 12 months after the initial incident). However, late chelation did not result in significant symptom relief.

Twenty-six workers exhibited symptoms related to erethism. Some also showed typical symptoms associated with cognitive mercury poisoning such as memory and attention disturbances $[23,24]$. Tremor of the hands, head, and eyelids, a late symptom of mercury poisoning, also occurred in some patients. EMG showed signs of mixed sensorimotor polyneuropathy and multiple mono-neuropathy alterations 12 to 18 months after exposure.

In this series, the VA decreased minimally and occurred in nine patients from G1 and five from G2; however, advanced visual functions were impaired significantly apparently independently of the mercury levels since significant negative correlations were seen only among the BML, BCVA, and ffERG.

Color vision and CS impairment at high spatial frequencies also were found, with the most frequently observed color vision alteration in the blue-yellow range. These findings agreed with previous studies [25, 27-29].

The most prevalent VF pattern was concentric constriction (17 eyes, 29.3\%), which agreed with previous studies [30, 31]. This visual impairment may have a central origin (calcarine cortex), as it has been reported previously [32]. In addition, the increased implicit time of P100 in the affected patients, especially in G3, indicates delayed nerve conduction and involvement of the visual pathway. da Costa et al. also reported this finding in 2008 (Costa et al. 2008). In the current series, significant retinal involvement also was seen, since the same patients showed retinal dysfunction in the ffERG, PERG, and mfERG tests, with both a generalized retinal response loss and alteration of the central retinal area, which could have affected the results obtained in the VF tests.

The ffERG showed changes in the SRR and OP of the ISCEV protocol, suggesting that rod cells are impaired by acute mercury-vapor intoxication. We did not find differences in the MSR, 30-Hz flicker, and SFCR data, which are key for assessing macular cone function, in the global sample compared to controls; however, significantly lower measurements were found in the ffERG in G3 compared to the controls, together with a lower amplitude of P50 in the PERG, suggesting that the cone cells and ganglion macular cells can be affected by mercury poisoning. These findings reinforce the idea that both the outer and inner retina visual processes are involved. Finally, the mfERG results are further evidence of damage to the photoreceptor pathway in mercury poisoning, since the amplitudes showed loss of the retinal response within the central 50 degrees, as reported previously [6]. 
A discrepancy was observed between the dysfunction patterns observed in the VF and the MfERG, with less involvement in the mfERG. This finding suggested retinal damage (detected by the $\mathrm{mfERG}$ ) in addition to that in the visual pathway.

The latency and amplitude of PRPEV were not correlated with the BML; however, patients in G3 had latencies significantly over 100 milliseconds and significantly reduced P100 amplitudes. Although these results typically occur in optic neuropathies and visual cortex abnormalities, they also can be associated with maculopathies, especially when they are interpreted in conjunction with retinal function tests (PERG, mfERG, and ffERG). These results agree with the results reported by Ventura et al. and da Costa et al. in patients with mercury poisoning $[6,19]$.

Despite the functional retinal involvement and in contrast to the results obtained by Ekinci et al [7, 8], OCT did not reveal structural changes in the RNFL, macular CRT, and choroid thickness when results were compared to the normalized reference values $[17,18]$. These differences might be related to the intensity and the manner of poisoning, as the current patients reached higher levels of mercury in a short time compared to the long exposure times of workers examined by Ekinci et al $[7,8]$.

This study has some limitations. There were no environmental measurements of mercury either before the accident or during the occupational incident. In addition, probably only the most affected patients were evaluated at the IOBA-Eye Institute, and the time that elapsed after the acute accident and the assessment at the IOBA likely was not the most appropriate for adequate follow-up over time. Most of the identified visual alterations, in our opinion, were attributable to the occupational exposure to mercury vapor, but we do not know the ophthalmologic baseline status before the accident. In addition, because of the lack of programmed follow-up, we had no information about the current clinical situation or about the evolution of most patients. Regarding the electrophysiologic tests, since half of the contaminated patients presented with no VA alterations, the results obtained from the global sample probably are affected by this patient subgroup. For this reason, our attention was focused on the subgroup G3. In addition, caution should be exercised when interpreting the results obtained from the comparisons between the control group and subgroup G3, because the samples size might be small for both groups in some comparisons. Finally, the OCT technology has evolved so rapidly that it is possible that with OCTs based on swept-source or ultra-high resolution it would have been possible to detect changes in the retinal or choroidal structures.

Even so, this study presents some relevant findings from a very rare and extremely serious event, for which references are scarce. First, the VA is affected slightly and there is more VF involvement. However, other visual function assessment tests seem to behave independently of the mercury levels. The most prevalent VF alteration is decreased VFs, but central involvement also was found. This finding could be of retinal and/or neurologic origin considering the mfERG results. No anatomic retinal changes were identified in this series, but it is possible that the new OCT systems allow establishing the structural bases of these alterations. Delayed chelation apparently did not benefit the patients.

In summary, despite its limitations, this series of patients affected by the same event contributes to the information obtained about mercury poisoning for future similar situations.

\section{Conclusions}

This is one of the largest series of mercury poissoning reported in the last years in which patients could be analyzed with new diagnostic techniques. Besides it is the only one reporting data on OCT or multifocal ERG after acute exposure to high concentrations mercury, as the current reports are on patients suffering chronic expposure. Finally, findings in the multifocal ERG allowed us to demonstrate that visual impairment after acute events is not only due to high visual pathway neurologic damage, but to retinal damage too at least in patients with the worst visual field outcomes.

\section{Declarations}

\section{Conflict of interest:}

All authors certify that they have no affiliations with or involvement in any organization or entity with any financial interest (such as honoraria; educational grants; participation in speakers' bureaus; membership, employment, consultancies, stock ownership, or other equity interest; and expert testimony or patent-licensing arrangements), or non-financial interest (such as personal or professional relationships, affiliations, knowledge or beliefs) in the subject matter or materials discussed in this manuscript.

\section{Ethical approval:}


All procedures performed were in accordance with the

ethical standards of the institutional and/or national research committee

and with the 1964 Helsinki declaration and its later amendments or comparable

ethical standards.

\section{Informed consent:}

Informed consent was obtained from all individual

participants included in the study.

\section{Funding sources:}

No funding was received for this research.

\section{Availability of data and material:}

All data and materials are available upon request.

\section{Author Contributions:}

AD-L, JLP-C and JCP conceived the study. AD-L and JLP-C collected and interpreted the systemic clinical data. JCP, IZ, YL, RC, JAdL and AM collected and analyzed the ophthamological clinical data. RMC-M interpreted the electrophysiological results. JCP analyzed the results all together. IF performed the statistical analysis. SP-I wrote the manuscript. All authors ensured that questions related to the accuracy or integrity of any part of the work were appropriately investigated, read and revised the final manuscript.

\section{Acknowledgments:}

Not applicable.

\section{References}

1. Satoh H. Occupational and environmental toxicology of mercury and its compounds. Ind Health. 2000;38:153-64; doi: 10.2486/indhealth.38.153.

2. Clarkson TW, Magos L, Myers GJ. The toxicology of mercury-current exposures and clinical manifestations. N Engl J Med. 2003;349:1731-7; doi: 10.1056/NEJMra022471.

3. Khayat A, Dencker L. Organ and cellular distribution of inhaled metallic mercury in the rat and the Marmoset monkey (Callithrix jacchus): influence of ethyl alcohol pretreatment. Acta Pharmacol Toxicol. 1984;55:145-52; doi: 10.1111/j.1600-

0773.1984.tb01977.x.

4. Warfvinge K, Bruun A. Mercury accumulation in the squirrel monkey eye after mercury vapour exposure. 1996;107:189-200; doi: 10.1016/0300-483x(95)03257-g.

5. Warfvinge K, Bruun A. Mercury distribution in the squirrel monkey retina after utero exposure to mercury vapor. Environ Res. 2000;83:102-9; doi: 10.1006/enrs.1999.4029.

6. Ventura DF, Costa MT, Costa MF, Berezovsky A, Salomão SR, Simões AL, et al. Multifocal and full-field electroretinogram changes associated with color-vision loss in mercury vapor exposure. Vis Neurosci. 2004;2:421-9; doi: 10.1017/s0952523804213372.

7. Ekinci M, Ceylan E, Keleş S, Cağatay HH, Apil A, Tanyıldız B, et al. Toxic effects of chronic mercury exposure on the retinal nerve fiber layer and macular and choroidal thickness in industrial mercury battery workers. Med Sci Monit. 2014;20:1284-90. doi: 10.12659/MSM.890756.

Page 15/17 
8. Ekinci M, Ceylan E, Cağatay HH, Keleş S, Altınkaynak H, Kartal B, et al. Occupational exposure to lead decreases macular, choroidal, and retinal nerve fiber layer thickness in industrial battery workers. Curr Eye Res. 2014;39:853-8; doi:

10.3109/02713683.2013.877934.

9. Sue YJ. Mercury. In: Nelson LS, Lewin NA, Howlan MA, Hoffman RS, Goldfrank LR, Flomenbaum NE eds. Goldfrank's Toxicological Emergencies $9^{\text {th }}$ New York: McGraw-Hill; 2011. p. 1299-1307.

10. Queipo Abad S, Rodríguez-González P, García Alonso Jl. Evidence of the direct adsorption of mercury in human hair during occupational exposure to mercury vapour. J Trace Elem Med Biol. 2016;36:16-21. doi: 10.1016/j.jtemb.2016.03.012.

11. Bowman KJ. A method for quantitative scoring of the Farnsworth Panel D-15. Acta Ophthalmol (Copenh). 1982;60:907-16; doi: 10.1111/j.1755-3768.1982.tb00621.x.

12. Vingrys AJ, King-Smith PE. A quantitative scoring technique for panel tests of color vision. Invest Ophthalmol Vis Sci. 1988;29:5063. PMID: 3257208.

13. WEB-based scoring software for the Farnsworth-Munsell 100-Hue, Roth 28-Hue, Farnsworth D-15, and the Lanthony D-15 desaturated color tests. Last change January 2014. http://www.belatorok.com/colorvision. Accessed 26 October 2020.

14. McCulloch DL, Marmor MF, Brigell MG, Hamilton R, Holder GE, Tzekov R, et al. ISCEV Standard for full-field clinical electroretinography (2015 update). Doc Ophthalmol Adv Ophthalmol. 2015;130:1-12; doi: 10.1007/s10633-014-9473-7.

15. International Society for Clinical Electrophysiology of Vision: Standards, Guidelines and Extended Protocols. https://iscev.wildapricot.org/standards. Accessed 26 October

16. Maturi KR, Yu M, Weleber RG. Multifocal electroretinographic evaluation of long term hydroxychloroquine users. Arch Ophthalmol. 2004;122: 973-81; doi: 10.1001/archopht.122.7.973.

17. TOPCON 3D OCT Series Normative Database. http://www.topconmedical.com/_assets/cf/downloadFile.cfm? section=productLit\&file=3D-OCT-Series-Normative-Summary.pdf\&parentUUID=55E1DBB5-CB99-9BF1-88CBBBE970ECAB77. Accessed 26 October 2020.

18. Kiernan DF, Hariprasad SM. Normative Databases in SD-OCT: A Status Report. http://www.retinalphysician.com/articleviewer.aspx? articlelD=10443. Accessed 26 October 2020.

19. da Costa GM, Anjos LM dos, Souza GS, Gomes BD, Saito CA, Pinheiro MCN, et al. Mercury toxicity in Amazon gold miners: visual dysfunction assessed by retinal and cortical electrophysiology. Environ Res. 2008;107:98-107; doi: 10.1016/j.envres.2007.08.004.

20. Nebbioso M, Livani ML, Steigerwalt RD, Panetta V, Rispoli E. Retina in rheumatic diseases: standard full field and multifocal electroretinography in hydroxychloroquine retinal dysfunction. Clin Exp Optom. 2011;94:276-83. doi: 10.1111/j.14440938.2010.00476.x.

21. Hood DC, Zhang X. Multifocal ERG and VEP responses and visual fields: comparing disease-related changes. Doc Ophthalmol. 2000;100:115-37; doi: 10.1023/a:1002727602212.

22. Falkenstein I, Kozak I, Kayikcioglu O, Cheng L, Bartsch DU, Azen SP, et al. Assessment of retinal function in patients with HIV without infectious retinitis by multifocal electroretinogram and automated perimetry. 2006;26:928-34; doi: 10.1097/01.iae.0000250009.60908.35

23. Takahata N, Hayashi H, Watanabe S, Anso T. Accumulation of mercury in the brains of two autopsy cases with chronic inorganic mercury poisoning. Folia Psychiatr Neurol Jpn. 1970;24:59-69; doi: 10.1111/j.1440-1819.1970.tb01457.x.

24. Asano S, Eto K, Kurisaki E, Gunji H, Hiraiwa K, Sato M, et al. Review article: acute inorganic mercury vapor inhalation poisoning. Pathol Int. 2000;50:169-74. doi: 10.1046/j.1440-1827.2000.01032.x.

25. Feitosa-Santana C, Costa MF, Lago M, Ventura DF. Long-term loss of color vision after exposure to mercury vapor. Braz J Med Biol Res. 2007;40: 409-14; doi: 10.1590/s0100-879x2007000300017.

26. Aslan L, Aslankurt M, Bozkurt S, Aksoy A, Ozdemir M, Gizir H, et al. Ophthalmic findings in acute mercury poisoning in adults: A case series study. Toxicol Ind Health. 2015;31:691-5; doi: 10.1177/0748233713483189.

27. Urban P, Gobba F, Nerudová J, Lukás E, Cábelková Z, Cikrt M. Color discrimination impairment in workers exposed to mercury vapor. 2003;24:711-6; doi: 10.1016/S0161-813X(03)00036-6.

28. Cavalleri A, Belotti L, Gobba F, Luzzana G, Rosa P, Seghizzi P. Colour vision loss in workers exposed to elemental mercury vapour. Toxicol Lett. 1995;77:351-6; doi: 10.1016/0378-4274(95)03317-3.

29. Costa MF, Tomaz S, de Souza JM, Silveira LC, Ventura DF. Electrophysiological evidence for impairment of contrast sensitivity in mercury vapor occupational intoxication. Environ Res. 2008;107:132-8; doi: 10.1016/j.envres.2007.10.007. 
30. Harada M. Minamata disease-methylmercury poisoning in Japan caused by environmental-pollution. Crit Rev Toxicol. 1995;25:1-24. doi: 10.3109/10408449509089885.

31. Barboni MT, da Costa MF, Moura AL, Feitosa-Santana C, Gualtieri M, Lago M, et al. Visual field losses in workers exposed to mercury vapor. Environ Res. 2008;107:24-131; doi: 10.1016/j.envres.2007.07.004.

32. Korogi Y, Takahashi M, Hirai T, Ikushima I, Kitajima M, Sugahara T, et al. Representation of the visual field in the striate cortex: comparison of MR findings with visual field deficits in organic mercury poisoning (Minamata disease). AJNR Am J Neuroradiol. 1997;18:1127-30; PMID: 9194440.

33. Stetson DS, Albers JW, Silverstein BA, Wolfe RA. Effects of age, sex, and anthropometric factors on nerve conduction measures. Muscle Nerve. 1992;15:1095-104; doi: 10.1002/mus.880151007.

34. Sedano MJ, Canga A, de Pablos C, Polo JM, Berciano J. Muscle MRI in severe Guillain-Barré syndrome with motor nerve inexcitability. J Neurol.2013;260:1624-30; doi: 10.1007/s00415-013-6845-y.

\section{Figures}

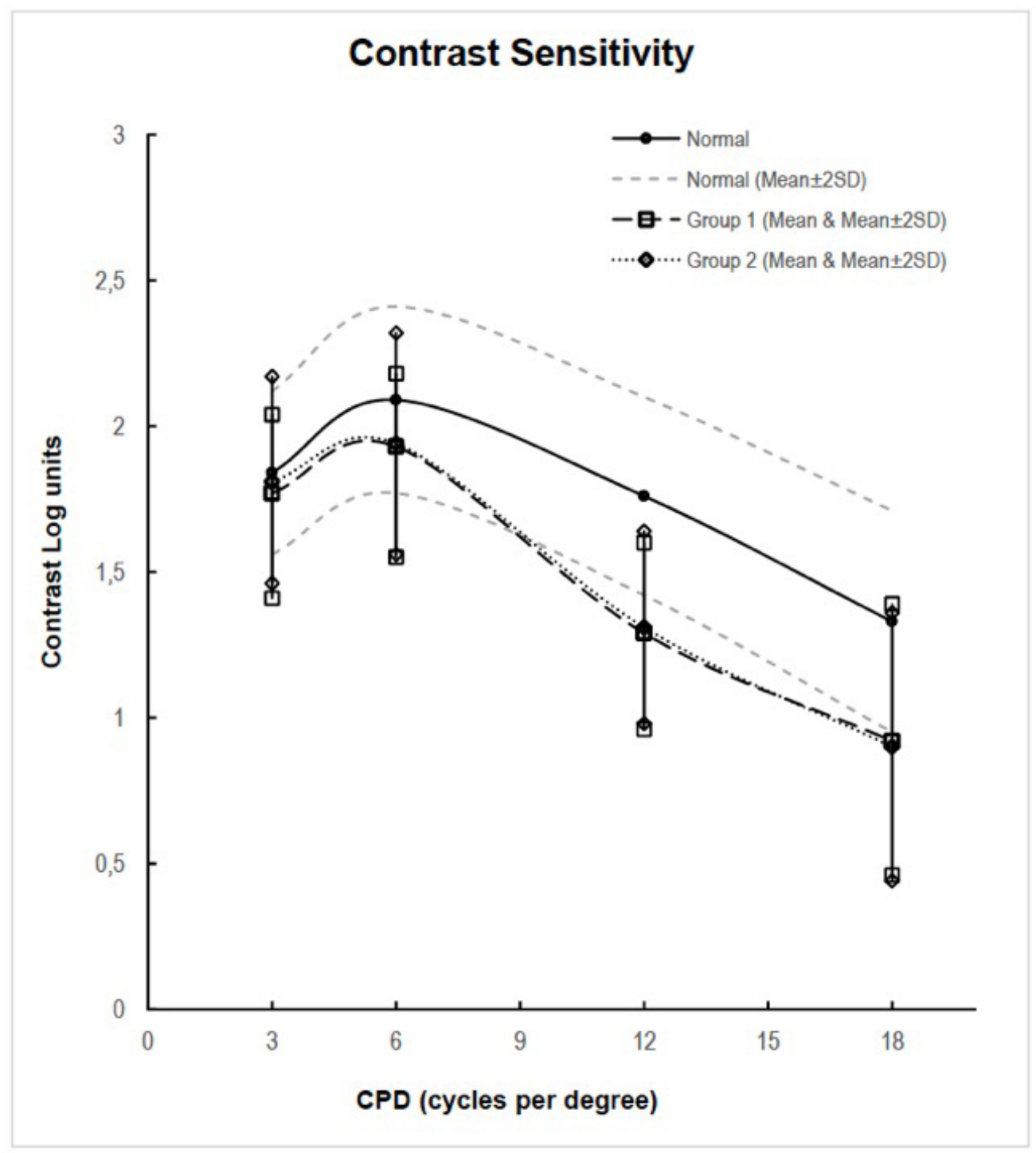

Figure 1

Average contrast sensitivity for two groups (G1 and G2) plotted as a function of spatial frequency. The squares and diamonds represent the average sensitivities for both groups at CS3, CS6, CS12 and CS18 respectively. Error bars represent \pm 2 SD of the mean. The area within the dashed lines represents the $95 \%$ interval of confidence for the normal population. SD=standard deviation. 